\title{
A Boundary Value Problem with Multivariables Integral Type Condition for Parabolic Equations
}

\author{
A. L. Marhoune ${ }^{1}$ and F. Lakhal ${ }^{2}$ \\ ${ }^{1}$ Laboratory Equations Différentielles, Departement of Mathematics, University Mentouri Constantine, \\ Constantine 25017, Algeria \\ ${ }^{2}$ Department of Mathematics, Science University of 08 Mai 45, P.O. Box 401, Guelma 24000, Algeria
}

Correspondence should be addressed to A. L. Marhoune, ahmed_marhoune@hotmail.com

Received 27 January 2009; Revised 14 May 2009; Accepted 13 October 2009

Recommended by Sergiu Aizicovici

We study a boundary value problem with multivariables integral type condition for a class of parabolic equations. We prove the existence, uniqueness, and continuous dependence of the solution upon the data in the functional wieghted Sobolev spaces. Results are obtained by using a functional analysis method based on two-sided a priori estimates and on the density of the range of the linear operator generated by the considered problem.

Copyright (c) 2009 A. L. Marhoune and F. Lakhal. This is an open access article distributed under the Creative Commons Attribution License, which permits unrestricted use, distribution, and reproduction in any medium, provided the original work is properly cited.

\section{Introduction}

Certain problems of modern physics and technology can be effectively described in terms of nonlocal problems with integral conditions for partial differential equations. These nonlocal conditions arise mainly when the data on the boundary cannot be measured directly. Motivated by this, we consider in the rectangular domain $\Omega=(0,1) \times(0, T)$, the following nonclassical boundary value problem of finding a solution $u(x, t)$ such that

$$
\mathcal{L} u=\frac{\partial u}{\partial t}-a(t) \frac{\partial^{2} u}{\partial x^{2}}=f(x, t)
$$

where the function $a(t)$ and its derivative are bounded on the interval $[0, T]$ :

$$
0<c_{0} \leq a(t) \leq c_{1}, \quad 0<c_{2} \leq \frac{d a(t)}{d t} \leq c_{3}
$$




$$
\begin{gathered}
l u=u(x, 0)=\varphi(x), \quad x \in(0,1), \\
u(0, t)=u(\beta, t)=u(\gamma, t)=u(1, t), \quad t \in(0, T), \\
\int_{0}^{\alpha} u(x, t) d x+2 \int_{\beta}^{\gamma} u(x, t) d x+\int_{\delta}^{1} u(x, t) d x=0, \\
0<\alpha<\beta<\gamma<\delta<1, \quad \alpha=1-\delta=\gamma-\beta, t \in(0, T) .
\end{gathered}
$$

Here, we assume that the known function $\varphi$ satisfies the conditions given in (1.4) and (1.5), that is,

$$
\varphi(0)=\varphi(\beta)=\varphi(\gamma)=\varphi(1), \quad \int_{0}^{\alpha} \varphi(x) d x+2 \int_{\beta}^{\gamma} \varphi(x) d x+\int_{\delta}^{1} \varphi(x) d x=0 .
$$

When considering the classical solution of the problem (1.1)-(1.5), along with (1.5), there should be the fulfilled conditions:

$$
\begin{gathered}
a(0)\left\{\varphi^{\prime \prime}(0)+\varphi^{\prime \prime}(\beta)-\varphi^{\prime \prime}(\gamma)-\varphi^{\prime \prime}(1)\right\}=f(1,0)+f(\gamma, 0)-f(\beta, 0)-f(0,0), \\
a(0)\left\{\int_{0}^{\alpha} \varphi^{\prime \prime}(x) d x+2 \int_{\beta}^{\gamma} \varphi^{\prime \prime}(x) d x+\int_{\delta}^{1} \varphi^{\prime \prime}(x) d x\right\}=\int_{0}^{\alpha} f(x, 0) d x+2 \int_{\beta}^{\gamma} f(x, 0) d x+\int_{\delta}^{1} f(x, 0) d x .
\end{gathered}
$$

Mathematical modelling of different phenomena leads to problems with nonlocal or integral boundary conditions. Such a condition occurs in the case when one measures an averaged value of some parameter inside the domain. This amounts to the specification of the energy or mass contained in a portion of the conductor or porous medium as a function of time. This problems arise in plasma physics, heat conduction, biology and demography, as well as modelling of technological process, see, for example, [1-5]. Boundary-value problems for parabolic equations with integral boundary condition are investigated by Batten [6], Bouziani and Benouar [7], Cannon [8, 9], Cannon, Perez Esteva and van der Hoek [10], Ionkin [11], Kamynin [12], Shi and Shillor [13], Shi [4], Marhoune and Bouzit [14], Marhoune and Hameida [15], Yurchuk [16], and many references therein. The problem with onevariable (resp., two-variable) boundary integral type condition is studied in [5] and by Marhoune and Latrous [17] (resp., in Marhoune [2]).

Mention that in the cited paper [16], the author proved the existence, uniqueness, and continuous dependence of a stronge solution in weighted Sobolev spaces to the problem

$$
\frac{\partial u}{\partial t}=\frac{\partial}{\partial x}\left(a(x, t) \frac{\partial u}{\partial x}\right)+f(x, t)
$$


under the following conditions:

$$
\begin{gathered}
u(x, 0)=0, \quad 0 \leq x \leq 1, \\
u(0, t)=0, \quad 0<t \leq T, \\
\int_{0}^{1} u(x, t) d x=0 .
\end{gathered}
$$

This last integral condition in the form

$$
\int_{0}^{1} u(x, t) d x=m(t), \quad 0<t \leq T
$$

arises, for example, in biochemistry in which $m$ is a constant, and in this case is known as the conservation of protein [18]. Further, in [5], the author studied a similar problem with the weak integral condition

$$
\int_{0}^{\alpha} u(x, t) d x=0, \quad 0<\alpha<1
$$

The same problem with the new integral condition

$$
\int_{0}^{\alpha} u(x, t) d x+\int_{\beta}^{1} u(x, t) d x=0, \quad \alpha+\beta=1,
$$

was investigated in [2]. The present paper is an extension in the same direction. By constructing a suitable multiplicator, we will try to establish existence and uniqueness of solution of problem (1.1)-(1.5). Note that the multivariables integral type condition (1.5) is considerably much weaker and better than that used in [2]. In fact, some physical problems have motivated specialists to consider nonlocal integral condition (1.5), which tells us the integral total effect of the solution $u$ over several independent portions $[0, \alpha],[\beta, \gamma]$, and $[\delta, 1]$ of interval $I=(0,1)$ at certain time $t$ that give this effect over the entire or part of this interval.

We associate with (1.1)-(1.5) the operator $L=(\mathcal{L}, l)$, defined from $E$ into $F$, where $E$ is the Banach space of functions $u \in L_{2}(\Omega)$, satisfying (1.4) and (1.5), with the finite norm

$$
\begin{aligned}
\|u\|_{E}^{2}= & \int_{\Omega} \frac{p(x)}{3}\left[\left|\frac{\partial u}{\partial t}\right|^{2}+\left|\frac{\partial^{2} u}{\partial x^{2}}\right|^{2}\right] d x d t+\sup _{0 \leq t \leq T} \int_{0}^{1} \frac{p(x)}{2}\left|\frac{\partial u}{\partial x}\right|^{2} d x \\
& +\sup _{0 \leq t \leq T} \int_{0}^{\alpha}|u|^{2} d x+\sup _{0 \leq t \leq T} \int_{\beta}^{\gamma}|u|^{2} d x+\sup _{0 \leq t \leq T} \int_{\delta}^{1}|u|^{2} d x
\end{aligned}
$$


and $F$ is the Hilbert space of vector-valued functions $\mathcal{F}=(f, \varphi)$ obtained by completion of the space $L_{2}(\Omega) \times W_{2}^{2}(0,1)$ with respect to the norm

$$
\|\mathscr{F}\|_{F}^{2}=\|(f, \varphi)\|_{F}^{2}=\int_{\Omega} \frac{p(x)}{3}|f|^{2} d x d t+\int_{0}^{1} \frac{p(x)}{2}\left|\frac{d \varphi}{d x}\right|^{2} d x+\int_{0}^{\alpha}|\varphi|^{2} d x+\int_{\beta}^{\gamma}|\varphi|^{2} d x+\int_{\delta}^{1}|\varphi|^{2} d x,
$$

where

$$
p(x)= \begin{cases}x^{2}, & x \in] 0, \alpha], \\ (\gamma-\beta)^{2}, & x \in[\alpha, \beta] \cup[\gamma, \delta], \\ (\gamma-x)^{2}+(x-\beta)^{2}, & x \in[\beta, \gamma], \\ (1-x)^{2}, & x \in[\delta, 1[.\end{cases}
$$

Using the energy inequalities method proposed in [16], we establish two-sided a priori estimates. Then, we prove that the operator $L$ is a linear homeomorphism between the spaces $E$ and $F$.

\section{Two-Sided A Priori Estimates}

Theorem 2.1. For any function $u \in E$, one has the a priori estimate

$$
\|L u\|_{F}^{2} \leq c_{4}\|u\|_{E}^{2}
$$

where the constant $c_{4}$ is independent of $u$. In fact, $c_{4}=2 \max \left(1, c_{1}^{2}\right)$.

Proof. Using (1.1) and initial condition (1.3), we obtain

$$
\begin{gathered}
\int_{\Omega} \frac{p(x)}{3}|\mathcal{L} u|^{2} d x d t \leq 2 \int_{\Omega} \frac{p(x)}{3}\left[\left|\frac{\partial u}{\partial t}\right|^{2}+c_{1}^{2}\left|\frac{\partial^{2} u}{\partial x^{2}}\right|^{2}\right] d x d t, \\
\int_{0}^{1} \frac{p(x)}{2}\left|\frac{d \varphi}{d x}\right|^{2} d x \leq \sup _{0 \leq t \leq T} \int_{0}^{1} \frac{p(x)}{2}\left|\frac{\partial u}{\partial x}\right|^{2} d x, \quad \int_{0}^{\alpha}|\varphi|^{2} d x \leq \sup _{0 \leq t \leq T} \int_{0}^{\alpha}|u|^{2} d x, \\
\int_{\beta}^{\gamma}|\varphi|^{2} d x \leq \sup _{0 \leq t \leq T} \int_{\beta}^{\gamma}|u|^{2} d x, \quad \int_{\delta}^{1}|\varphi|^{2} d x \leq \sup _{0 \leq t \leq T} \int_{\delta}^{1}|u|^{2} d x .
\end{gathered}
$$

Combining the inequalities in (2.2), we obtain (2.1) for $u \in E$. 
Journal of Applied Mathematics and Stochastic Analysis

Theorem 2.2. For any function $u \in E$, one has the a priori estimate

$$
\|u\|_{E}^{2} \leq c_{5}\|L u\|_{F}^{2}
$$

with the constant

$$
c_{5}=\frac{\exp (c T) \max \left(49,2 c_{1}\right)}{\min \left(13 / 32, c_{0}, c_{0}^{2} / 2\right)}
$$

and $c$ is such that

$$
c c_{0} \geq c_{3}
$$

Before proving this theorem, we need the following lemma.

Lemma 2.3 (see [19]). For $u \in E$, one has

$$
\begin{gathered}
\int_{a}^{b}\left|\int_{x}^{b} \frac{\partial u(\xi, t)}{\partial t} d \xi\right|^{2} d x \leq 4 \int_{a}^{b}(x-a)^{2}\left|\frac{\partial u}{\partial t}\right|^{2} d x \\
\int_{a}^{b}\left|\int_{a}^{x} \frac{\partial u(\xi, t)}{\partial t} d \xi\right|^{2} d x \leq 4 \int_{a}^{b}(b-x)^{2}\left|\frac{\partial u}{\partial t}\right|^{2} d x .
\end{gathered}
$$

Proof of Theorem 2.2. Define

$$
M u= \begin{cases}x^{2} \frac{\partial u}{\partial t}+2 x \int_{x}^{\alpha} \frac{\partial u(\xi, t)}{\partial t} d \xi, & x \in] 0, \alpha] \\ (\gamma-\beta)^{2} \frac{\partial u}{\partial t}, & x \in[\alpha, \beta] \cup[\gamma, \delta], \\ (\gamma-x)^{2} \frac{\partial u}{\partial t}+(x-\beta)^{2} \frac{\partial u}{\partial t}+2(\gamma-x) \int_{\beta}^{x} \frac{\partial u(\xi, t)}{\partial t} d \xi & \\ +2(x-\beta) \int_{x}^{\gamma} \frac{\partial u(\xi, t)}{\partial t} d \xi, & x \in[\beta, \gamma] \\ (1-x)^{2} \frac{\partial u}{\partial t}+2(1-x) \int_{\delta}^{x} \frac{\partial u(\xi, t)}{\partial t} d \xi, & x \in[\delta, 1[.\end{cases}
$$

We consider for $u \in E$ the quadratic formula

$$
\operatorname{Re} \int_{0}^{\tau} \int_{0}^{1} \exp (-c t) \mathcal{L} \overline{M u} d x d t
$$


with the constant $c$ satisfying (2.5), obtained by multiplying (1.1) by $\exp (-c t) M u$, by integrating over $\Omega^{\tau}$, where $\Omega^{\tau}=(0,1) \times(0, \tau)$, with $0 \leq \tau \leq T$, and by taking the real part. Integrating by parts in (2.8) by report to $x$ with the use of boundary conditions (1.4) and (1.5), we obtain

$$
\begin{aligned}
& \operatorname{Re} \int_{0}^{\tau} \int_{0}^{1} \exp (-c t) \mathcal{L} \overline{M u} d x d t \\
& =\int_{0}^{\tau} \int_{0}^{1} p(x) \exp (-c t)\left|\frac{\partial u}{\partial t}\right|^{2} d x d t+\int_{0}^{\tau} \int_{0}^{\alpha} \exp (-c t)\left|\int_{x}^{\alpha} \frac{\partial u(\xi, t)}{\partial t} d \xi\right|^{2} d x d t \\
& \quad+\int_{0}^{\tau} \int_{\beta}^{\gamma} \exp (-c t)\left[\left|\int_{\beta}^{x} \frac{\partial u(\xi, t)}{\partial t} d \xi\right|^{2}+\left|\int_{x}^{\gamma} \frac{\partial u(\xi, t)}{\partial t} d \xi\right|^{2}\right] d x d t \\
& \quad+\int_{0}^{\tau} \int_{\delta}^{1} \exp (-c t)\left|\int_{\delta}^{x} \frac{\partial u(\xi, t)}{\partial t} d \xi\right|^{2} d x d t+\operatorname{Re} \int_{0}^{\tau} \int_{0}^{1} \exp (-c t) p(x) a \frac{\partial u}{\partial x} \frac{\partial^{2} \bar{u}}{\partial x \partial t} d x d t \\
& \quad+2 \operatorname{Re} \int_{0}^{\tau} \int_{0}^{\alpha} \exp (-c t) a u \frac{\partial \bar{u}}{\partial t} d x d t+4 \operatorname{Re} \int_{0}^{\tau} \int_{\beta}^{\gamma} \exp (-c t) a u \frac{\partial \bar{u}}{\partial t} d x d t \\
& \quad+2 \operatorname{Re} \int_{0}^{\tau} \int_{\delta}^{1} \exp (-c t) a u \frac{\partial \bar{u}}{\partial t} d x d t .
\end{aligned}
$$

On the other hand, by using the elementary inequalities we get

$$
\begin{aligned}
& \operatorname{Re} \int_{0}^{\tau} \int_{0}^{1} \exp (-c t) \mathcal{\perp} \overline{M u} d x d t \\
& \geq \int_{0}^{\tau} \int_{0}^{1} p(x) \exp (-c t)\left|\frac{\partial u}{\partial t}\right|^{2} d x d t+\operatorname{Re} \int_{0}^{\tau} \int_{0}^{1} \exp (-c t) p(x) a \frac{\partial u}{\partial x} \frac{\partial^{2} \bar{u}}{\partial x \partial t} d x d t \\
& \quad+2 \operatorname{Re} \int_{0}^{\tau} \int_{0}^{\alpha} \exp (-c t) a u \frac{\partial \bar{u}}{\partial t} d x d t+4 \operatorname{Re} \int_{0}^{\tau} \int_{\beta}^{\gamma} \exp (-c t) a u \frac{\partial \bar{u}}{\partial t} d x d t \\
& \quad+2 \operatorname{Re} \int_{0}^{\tau} \int_{\delta}^{1} \exp (-c t) a u \frac{\partial \bar{u}}{\partial t} d x d t
\end{aligned}
$$


Again, integrating by parts the second, third, fourth, and fifth terms of the right-hand side of the inequality (2.10) by report to $t$ and taking into account the initial condition (1.3) and (2.5) gives

$$
\begin{aligned}
& \operatorname{Re} \int_{0}^{\tau} \int_{0}^{1} \exp (-c t) p(x) a \frac{\partial u}{\partial x} \frac{\partial^{2} \bar{u}}{\partial x \partial t} d x d t \geq \int_{0}^{1} \frac{\exp (-c \tau)}{2} p(x) a(\tau)\left|\frac{\partial u(x, \tau)}{\partial x}\right|^{2} d x \\
&-\frac{1}{2} \int_{0}^{1} p(x) a(0)\left|\frac{d l u}{d x}\right|^{2} d x, \\
& \operatorname{Re} \int_{0}^{\tau} \int_{0}^{\alpha} \exp (-c t) a u \frac{\partial \bar{u}}{\partial t} d x d t \geq \int_{0}^{\alpha} \frac{\exp (-c \tau)}{2} a(\tau)|u(x, \tau)|^{2} d x-\int_{0}^{\alpha} \frac{a(0)}{2}|l u|^{2} d x \\
& \operatorname{Re} \int_{0}^{\tau} \int_{\beta}^{\gamma} \exp (-c t) a u \frac{\partial \bar{u}}{\partial t} d x d t \geq \int_{\beta}^{\gamma} \frac{\exp (-c \tau)}{2} a(\tau)|u(x, \tau)|^{2} d x-\int_{\beta}^{\gamma} \frac{a(0)}{2}|l u|^{2} d x, \\
& \operatorname{Re} \int_{0}^{\tau} \int_{\delta}^{1} \exp (-c t) a u \frac{\partial \bar{u}}{\partial t} d x d t \geq \int_{\delta}^{1} \frac{\exp (-c \tau)}{2} a(\tau)|u(x, \tau)|^{2} d x-\int_{\delta}^{1} \frac{a(0)}{2}|l u|^{2} d x .
\end{aligned}
$$

Using (2.11) in (2.10), we get

$$
\begin{aligned}
& \operatorname{Re} \int_{0}^{\tau} \int_{0}^{1} \exp (-c t) \mathcal{L} \overline{M u} d x d t+\int_{0}^{\alpha} a(0)|l u|^{2} d x+2 \int_{\beta}^{\gamma} a(0)|l u|^{2} d x \\
& \quad+\int_{\delta}^{1} a(0)|l u|^{2} d x+\frac{1}{2} \int_{0}^{1} p(x) a(0)\left|\frac{d l u}{d x}\right|^{2} d x \\
& \geq \int_{0}^{\tau} \int_{0}^{1} p(x) \exp (-c t)\left|\frac{\partial u}{\partial t}\right|^{2} d x d t \\
& \quad+\frac{1}{2} \int_{0}^{1} \exp (-c \tau) p(x) a(\tau)\left|\frac{\partial u(x, \tau)}{\partial x}\right|^{2} d x+\int_{0}^{\alpha} \exp (-c \tau) a(\tau)|u(x, \tau)|^{2} d x \\
& \quad+2 \int_{\beta}^{\gamma} \exp (-c \tau) a(\tau)|u(x, \tau)|^{2} d x+\int_{\delta}^{1} \exp (-c \tau) a(\tau)|u(x, \tau)|^{2} d x
\end{aligned}
$$


By using the $\varepsilon$-inequalities on the first integral in the left-hand side of (2.12) and Lemma 2.3, we obtain

$$
\begin{aligned}
\frac{15}{32} \int_{0}^{\tau} \int_{0}^{1} p(x) \exp (-c t)\left|\frac{\partial u}{\partial t}\right|^{2} d x d t+\frac{1}{2} \int_{0}^{1} \exp (-c \tau) p(x) a(\tau)\left|\frac{\partial u(x, \tau)}{\partial x}\right|^{2} d x \\
\quad+\int_{0}^{\alpha} \exp (-c \tau) a(\tau)|u(x, \tau)|^{2} d x+2 \int_{\beta}^{\gamma} \exp (-c \tau) a(\tau)|u(x, \tau)|^{2} d x \\
\quad+\int_{\delta}^{1} \exp (-c \tau) a(\tau)|u(x, \tau)|^{2} d x \\
\leq 16 \int_{0}^{\tau} \int_{0}^{1} p(x) \exp (-c t)|\mathcal{L} u|^{2} d x d t \\
\quad+\int_{0}^{\alpha} a(0)|l u|^{2} d x+\frac{1}{2} \int_{0}^{1} p(x) a(0)\left|\frac{d l u}{d x}\right|^{2} d x+2 \int_{\beta}^{\gamma} a(0)|l u|^{2} d x+\int_{\delta}^{1} a(0)|l u|^{2} d x .
\end{aligned}
$$

Now, from (1.1), we have

$$
\begin{aligned}
& \frac{c_{0}^{2}}{6} \int_{0}^{\tau} \int_{0}^{1} p(x) \exp (-c t)\left|\frac{\partial^{2} u}{\partial x^{2}}\right|^{2} d x d t \\
& \quad \leq \int_{0}^{\tau} \int_{0}^{1} \frac{p(x)}{3} \exp (-c t)|\mathcal{L} u|^{2} d x d t+\int_{0}^{\tau} \int_{0}^{1} \frac{p(x)}{3} \exp (-c t)\left|\frac{\partial u}{\partial t}\right|^{2} d x d t .
\end{aligned}
$$

Combining inequalities (2.13) and (2.14), we get

$$
\begin{aligned}
& \exp (-c T)\left(\frac{13}{32} \int_{0}^{\tau} \int_{0}^{1} \frac{p(x)}{3}\left|\frac{\partial u}{\partial t}\right|^{2} d x d t+c_{0} \int_{0}^{1} \frac{p(x)}{2}\left|\frac{\partial u(x, \tau)}{\partial x}\right|^{2} d x+c_{0} \int_{0}^{\alpha}|u(x, \tau)|^{2} d x\right. \\
& \left.\quad+2 c_{0} \int_{\beta}^{\gamma}|u(x, \tau)|^{2} d x+c_{0} \int_{\delta}^{1}|u(x, \tau)|^{2} d x+\frac{c_{0}^{2}}{6} \int_{0}^{\tau} \int_{0}^{1} \frac{p(x)}{3}\left|\frac{\left.\partial^{2} u\right|^{2}}{\partial x^{2}}\right|^{2} d x d t\right) \\
& \leq 49 \int_{\Omega} \frac{p(x)}{3}|\mathcal{L} u|^{2} d x d t+c_{1} \int_{0}^{1} \frac{p(x)}{2}\left|\frac{d l u}{d x}\right|^{2} d x+c_{1} \int_{0}^{\alpha}|l u|^{2} d x+2 c_{1} \int_{\beta}^{\gamma}|l u|^{2} d x+c_{1} \int_{\delta}^{1}|l u|^{2} d x .
\end{aligned}
$$

As the right-hand side of (2.15) is independent of $\tau$, by replacing the left-hand side by its upper bound with respect to $\tau$ in the interval $[0, T]$, we obtain the desired inequality. 


\section{Solvability of the Problem}

From estimates (2.1) and (2.3), it follows that the operator $L: E \rightarrow F$ is continuous and its range is closed in $F$. Therefore, the inverse operator $L^{-1}$ exists and is continuous from the closed subspace $R(L)$ onto $E$, which means that $L$ is an homeomorphism from $E$ onto $R(L)$. To obtain the uniqueness of solution, it remains to show that $R(L)=F$. The proof is based on the following lemma.

Lemma 3.1. Let

$$
D_{0}(L)=\{u \in E: l u=0\} .
$$

If for $u \in D_{0}(L)$ and some $w \in L_{2}(\Omega)$, one has

$$
\int_{\Omega} q(x) \mathcal{u} \bar{w} d x d t=0
$$

where

$$
q(x)= \begin{cases}x, & x \in] 0, \alpha], \\ \gamma-\beta, & x \in[\alpha, \delta], \\ 1-x, & x \in[\delta, 1[\end{cases}
$$

then $w=0$.

Proof. From (3.2) we have

$$
\int_{\Omega} q(x) \frac{\partial u}{\partial t} \bar{w} d x d t=\int_{\Omega} q(x) a(t) \frac{\partial^{2} u}{\partial x^{2}} \bar{w} d x d t
$$

Now, for given $w(x, t)$, we introduce the function

$$
v(x, t)= \begin{cases}w(x, t)-\int_{x}^{\alpha} \frac{w(\xi, t)}{\xi} d \xi, & x \in] 0, \alpha], \\ w(x, t), & x \in[\alpha, \delta], \\ w(x, t)-\int_{\delta}^{x} \frac{w(\xi, t)}{\xi} d \xi, & x \in[\delta, 1[.\end{cases}
$$


Integrating by parts with respect to $\xi$, we obtain

$$
q(x) w= \begin{cases}x v+\int_{x}^{\alpha} v(\xi, t) d \xi, & x \in] 0, \alpha], \\ (\gamma-\beta) v, & x \in[\alpha, \beta] \cup[\gamma, \delta], \\ (\gamma-\beta) v+\int_{\beta}^{\gamma} v(\xi, t) d \xi, & x \in[\beta, \gamma], \\ (1-x) v+\int_{\delta}^{x} v(\xi, t) d \xi, & x \in[\delta, 1[\end{cases}
$$

which implies that

$$
\int_{0}^{\alpha} v(\xi, t) d \xi+2 \int_{\beta}^{\gamma} v(\xi, t) d \xi+\int_{\delta}^{1} v(\xi, t) d \xi=0
$$

Then, from (3.4), we obtain

$$
-\int_{\Omega} \frac{\partial u}{\partial t} \overline{N v} d x d t=\int_{\Omega} A(t) u \bar{v} d x d t
$$

where

$$
N v=q(x) v, \quad A(t) u=-\frac{\partial}{\partial x}\left(q(x) a(t) \frac{\partial u}{\partial x}\right)
$$

If we introduce the smoothing operators with respect to $t[16], \mathfrak{I}_{\varepsilon}^{-1}=(I+\varepsilon \partial / \partial t)^{-1}$ and $\left(\mathfrak{I}_{\mathcal{\varepsilon}}^{-1}\right)^{*}$, then these operators provide the solutions of the respective problems:

$$
\begin{aligned}
& \varepsilon \frac{d g_{\varepsilon}(t)}{d t}+g_{\varepsilon}(t)=g(t), g_{\varepsilon}(t)_{t=0}=0, \\
&-\varepsilon \frac{d g_{\varepsilon}^{*}(t)}{d t}+g_{\varepsilon}^{*}(t)=g(t), \quad g_{\varepsilon}^{*}(t)_{t=T}=0,
\end{aligned}
$$

and also have the following properties: for any $g \in L_{2}(0, T)$, the functions $g_{\varepsilon}=\left(\mathfrak{J}_{\varepsilon}^{-1}\right) g$ and $g_{\varepsilon}^{*}=\left(\mathfrak{I}_{\varepsilon}^{-1}\right)^{*} g$ are in $W_{2}^{1}(0, T)$ such that $g_{\varepsilon}(t)_{t=0}=0$ and $g_{\varepsilon}^{*}(t)_{t=T}=0$. Morever, $\mathfrak{I}_{\varepsilon}^{-1}$ commutes with $\partial / \partial t$, so $\int_{0}^{T}\left|g_{\varepsilon}-g\right|^{2} d t \rightarrow 0$ and $\int_{0}^{T}\left|g_{\varepsilon}^{*}-g\right|^{2} d t \rightarrow 0$ for $\varepsilon \rightarrow 0$.

Putting $u=\int_{0}^{t} \exp (c \tau) v_{\varepsilon}^{*}(x, \tau) d \tau$ in (3.8), where the constant $c$ satisfies $c c_{0}-c_{3}-$ $\varepsilon c_{3}^{2} / c_{0} \geq 0$, and using (3.11), we obtain

$$
-\int_{\Omega} \exp (c t) v_{\mathcal{\varepsilon}}^{*} \overline{N v} d x d t=\int_{\Omega} A(t) u \exp (-c t) \frac{\partial \bar{u}}{\partial t} d x d t-\varepsilon \int_{\Omega} A(t) u \frac{\partial \overline{v_{\varepsilon}^{*}}}{\partial t} d x d t
$$



yield

Integrating by parts each term in the right-hand side of (3.12) and taking the real parts

$$
\begin{aligned}
2 \operatorname{Re} \int_{\Omega} A(t) u \exp (-c t) \frac{\partial \bar{u}}{\partial t} d x d t= & \int_{0}^{1} a(T) q(x) \exp (-c T)\left|\frac{\partial u(x, T)}{\partial t}\right|^{2} d x \\
& +\int_{\Omega} q(x) \exp (-c t)\left(c a(t)-\frac{d a(t)}{d t}\right)\left|\frac{\partial u}{\partial x}\right|^{2} d x d t \\
\operatorname{Re}\left(-\varepsilon \int_{\Omega} A(t) u \frac{\partial \overline{v_{\varepsilon}^{*}}}{\partial t} d x d t\right)= & \operatorname{Re}\left(\varepsilon \int_{\Omega} \frac{d a(t)}{d t} q(x) \frac{\partial u}{\partial x} \frac{\partial \overline{v_{\varepsilon}^{*}}}{\partial x} d x d t\right) \\
& +\varepsilon \int_{\Omega} a(t) \exp (c t) q(x) \frac{\partial \overline{v_{\varepsilon}^{*}}}{\partial x} d x d t .
\end{aligned}
$$

Using $\varepsilon$-inequalities, we obtain

$$
\operatorname{Re}\left(-\varepsilon \int_{\Omega} A(t) u \frac{\partial \overline{v_{\varepsilon}^{*}}}{\partial t} d x d t\right) \geq-\frac{\varepsilon c_{3}^{2}}{2 c_{0}} \int_{\Omega} q(x) \exp (-c t)\left|\frac{\partial u}{\partial x}\right|^{2} d x d t
$$

Combining (3.13) and (3.15), we get

$$
\operatorname{Re}\left(\int_{\Omega} \exp (c t) v_{\varepsilon}^{*} \overline{N v} d x d t\right) \leq-\int_{\Omega} q(x) \exp (-c t)\left(c c_{0}-c_{3}-\frac{\varepsilon c_{3}^{2}}{c_{0}}\right)\left|\frac{\partial u}{\partial x}\right|^{2} d x d t \leq 0
$$

From (3.16), we deduce that

$$
\operatorname{Re}\left(\int_{\Omega} \exp (c t) v_{\varepsilon}^{*} \overline{N v} d x d t\right) \leq 0
$$

Then, for $\varepsilon \rightarrow 0$, we obtain

$$
\operatorname{Re} \int_{\Omega} \exp (c t) v \overline{N v} d x d t=\int_{\Omega} q(x) \exp (c t)|v|^{2} d x d t \leq 0
$$

We conclude that $v=0$, hence $w=0$, which ends the proof of the the lemma.

Theorem 3.2. The range $R(L)$ of $L$ coincides with $F$.

Proof. Since $F$ is a Hilbert space, we have $R(L)=F$ if and only if the relation

$$
\int_{\Omega} \frac{p(x)}{3} \mathcal{L} \bar{u} d x d t+\int_{0}^{1} \frac{p(x)}{2} \frac{d l u}{d x} \frac{d \bar{\varphi}}{d x} d x+\int_{0}^{\alpha} l u \bar{\varphi} d x+\int_{\beta}^{\gamma} l u \bar{\varphi} d x+\int_{\delta}^{1} l u \bar{\varphi} d x=0,
$$

for arbitrary $u \in E$ and $(f, \varphi) \in F$, implies that $f=0$ and $\varphi=0$. 
Putting $u \in D_{0}(L)$ in (3.19), we conclude from Lemma 3.1 that $\theta f=0$, where

$$
\theta f= \begin{cases}x f, & x \in] 0, \alpha], \\ (\gamma-\beta) f, & x \in[\alpha, \delta], \\ (1-x) f, & x \in[\delta, 1[\end{cases}
$$

then $f=0$.

Taking $u \in E$ in (3.19) yields

$$
\int_{0}^{1} \frac{p(x)}{2} \frac{d l u}{d x} \frac{d \bar{\varphi}}{d x} d x+\int_{0}^{\alpha} l u \bar{\varphi} d x+\int_{\beta}^{\gamma} l u \bar{\varphi} d x+\int_{\delta}^{1} l u \bar{\varphi} d x=0 .
$$

The range of the operator $l$ is everywhere dense in Hilbert space with the norm

$$
\left[\int_{0}^{1} \frac{p(x)}{2}\left|\frac{d \varphi}{d x}\right|^{2} d x+\int_{0}^{\alpha}|\varphi|^{2} d x+\int_{\beta}^{\gamma}|\varphi|^{2} d x+\int_{\delta}^{1}|\varphi|^{2} d x\right]^{1 / 2},
$$

hence, $\varphi=0$.

\section{Acknowledgment}

The authors would like to thank the referee for helpful suggestions and comments.

\section{References}

[1] A. A. Samarski1̌, "Some problems of the theory of differential equations," Differentsial'nye Uravneniya, vol. 16, no. 11, pp. 1221-1228, 1980.

[2] A. L. Marhoune, "A three-point boundary value problem with an integral two-space-variables condition for parabolic equations," Computers \& Mathematics with Applications, vol. 53, no. 6, pp. 940 947, 2007.

[3] R. E. Ewing and T. Lin, "A class of parameter estimation techniques for fluid flow in porous media," Advances in Water Resources, vol. 14, no. 2, pp. 89-97, 1991.

[4] P. Shi, "Weak solution to an evolution problem with a nonlocal constraint," SIAM Journal on Mathematical Analysis, vol. 24, no. 1, pp. 46-58, 1993.

[5] A. V. Kartynnik, "A three-point mixed problem with an integral condition with respect to the space variable for second-order parabolic equations," Differential Equations, vol. 26, no. 9, pp. 1160-1166, 1990.

[6] G. W. Batten Jr., "Second-order correct boundary conditions for the numerical solution of the mixed boundary problem for parabolic equations," Mathematics of Computation, vol. 17, pp. 405-413, 1963.

[7] A. Bouziani and N.-E. Benouar, "Mixed problem with integral conditions for a third order parabolic equation," Kobe Journal of Mathematics, vol. 15, no. 1, pp. 47-58, 1998.

[8] J. R. Cannon, "The solution of the heat equation subject to the specification of energy," Quarterly of Applied Mathematics, vol. 21, pp. 155-160, 1963.

[9] J. R. Cannon, The One-Dimensional Heat Equation, vol. 23 of Encyclopedia of Mathematics and Its Applications, Addison-Wesley, Mento Park, Calif, USA, 1984.

[10] J. R. Cannon, S. Pérez Esteva, and J. van der Hoek, "A Galerkin procedure for the diffusion equation subject to the specification of mass," SIAM Journal on Numerical Analysis, vol. 24, no. 3, pp. 499-515, 1987. 
[11] N. I. Ionkin, "The solution of a certain boundary value problem of the theory of heat conduction with a nonclassical boundary condition," Differentsial'nye Uravneniya, vol. 13, no. 2, pp. 294-304, 1977.

[12] L. I. Kamynin, "A boundary-value problem in the theory of heat conduction with non-classical boundary conditions," U.S.S.R. Computational Mathematics and Mathematical Physicsi, vol. 4, pp. 3359, 1964.

[13] P. Shi and M. Shillor, "Design of contact patterns in one dimentional thermoelasticity," in Theoretical Aspects of Industrial Design, SIAM, Philadelphia, Pa, USA, 1992.

[14] A. L. Marhoune and M. Bouzit, "High order differential equations with integral boundary condition," Far East Journal of Mathematical Sciences, vol. 18, no. 3, pp. 341-350, 2005.

[15] A. L. Marhoune and A. Hameida, "Mixed problem with an integral space variable condition for a third order parabolic equation of mixed type," Far East Journal of Mathematical Sciences, vol. 29, no. 2, pp. 409-418, 2008.

[16] N. I. Yurchuk, "Mixed problem with an integral condition for some parabolic equations," Differential Equations, vol. 22, pp. 1457-1463, 1986.

[17] A. L. Marhoune and C. Latrous, "A strong solution of a high-order mixed type partial differential equation with integral conditions," Applicable Analysis, vol. 87, no. 6, pp. 625-634, 2008.

[18] G. Fairweather and R. D. Saylor, "The reformulation and numerical solution of certain nonclassical initial-boundary value problems," SIAM Journal on Scientific and Statistical Computing, vol. 12, no. 1, pp. 127-144, 1991.

[19] G. H. Hardy, J. E. Littlewood, and G. Polya, Inequalities, Cambridge Press, Cambridge, UK, 1934. 


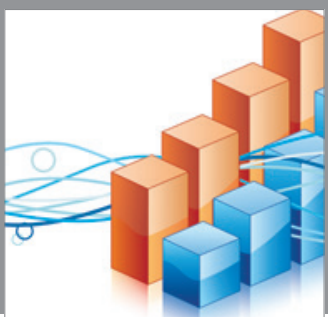

Advances in

Operations Research

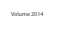

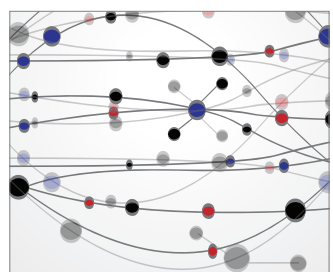

\section{The Scientific} World Journal
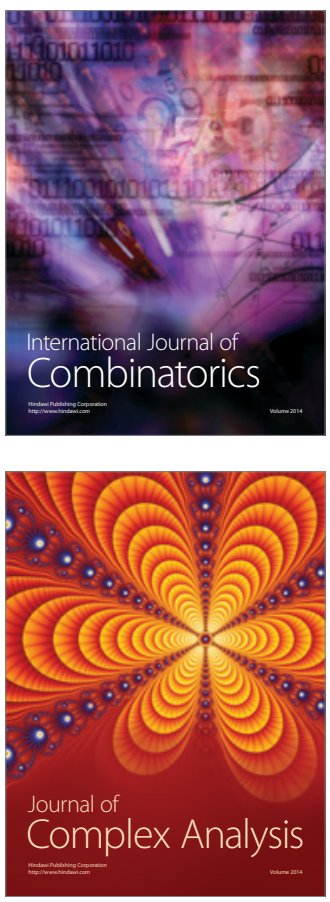

International Journal of

Mathematics and

Mathematical

Sciences
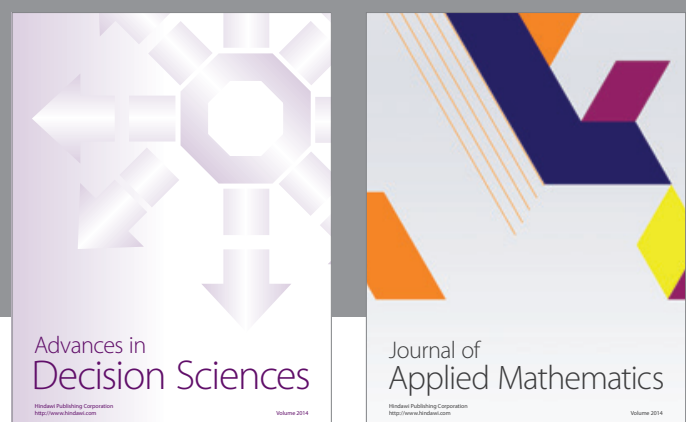

Journal of

Applied Mathematics
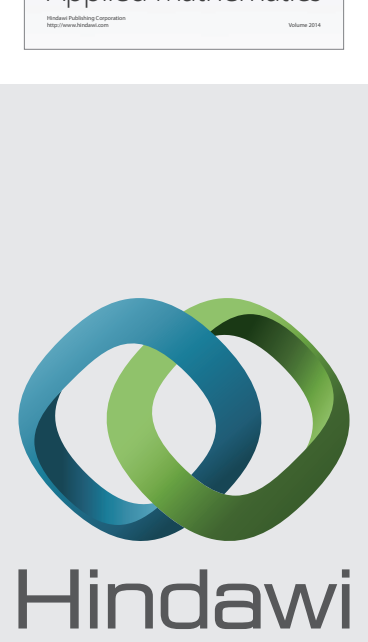

Submit your manuscripts at http://www.hindawi.com
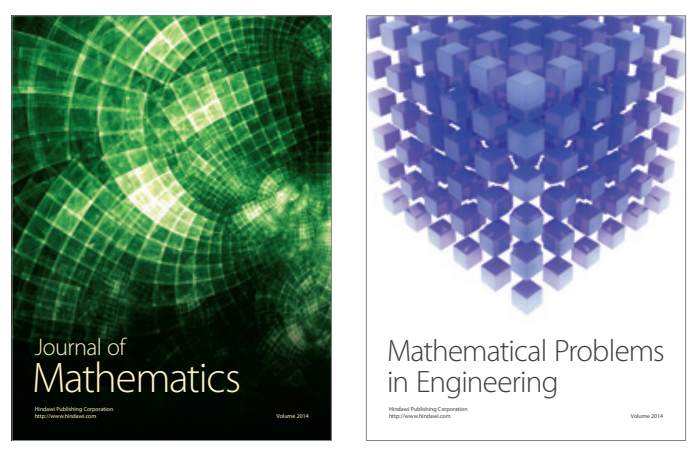

Mathematical Problems in Engineering
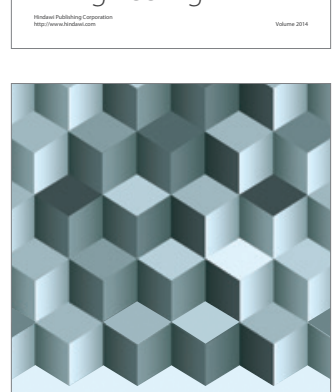

Journal of

Function Spaces
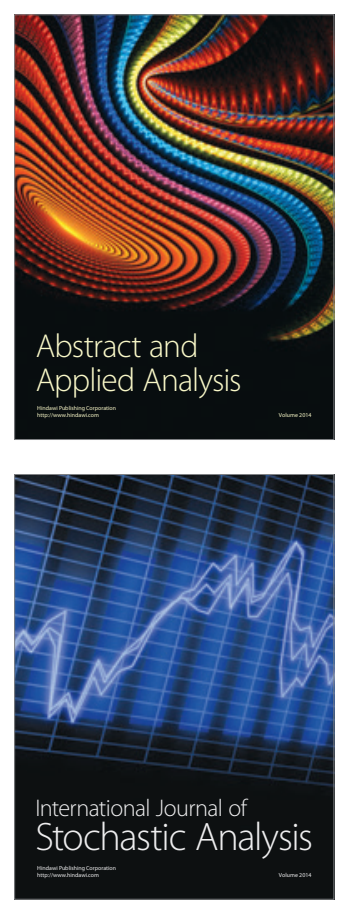

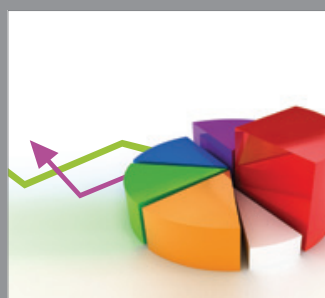

ournal of

Probability and Statistics

Promensencen
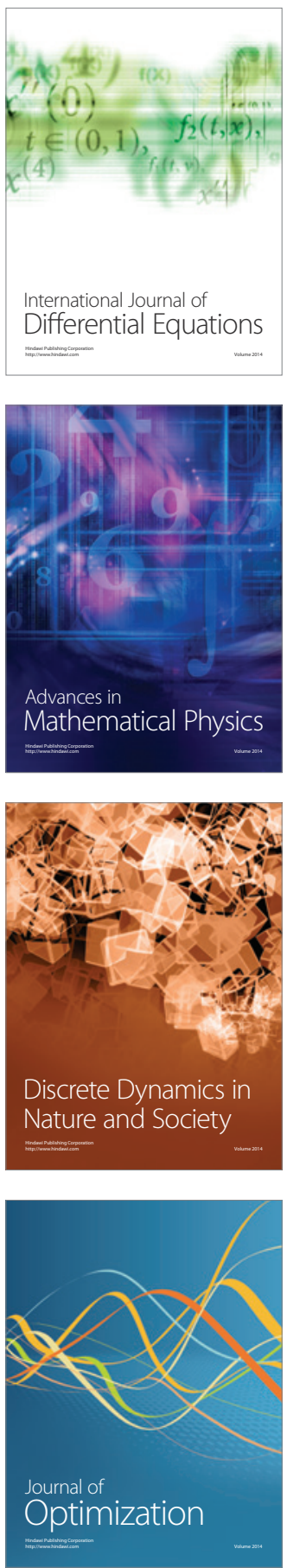